\title{
Herramientas tecnológicas para una virtualidad en la educación
}

\author{
Mg. María Amparo de Dios Ruiz Sánchez \\ rsanchezma@ucvvirtual.edu.pe \\ https://orcid.org/0000-0003-3061-3601
}

Mg. Nikar Tatiana Aguirre Gonzales

agonzalesn@ucvvirtual.edu.pe https://orcid.org/0000-0002-6084-6767

Dr. Luis Montenegro Camacho

mcamacholar@ucvvirtual.edu.pe https://orcid.org/0000-0002-8696-5203

\section{Mg. Oscar Darwin Mendoza Cruzado \\ mcruzadood@ucvvirtual.edu.pe \\ https://orcid.org/0000-0002-4891-726X}

Programa Académico de Doctorado en Educación

Escuela de postgrado

Universidad César Vallejo

Chiclayo - Perú

\section{RESUMEN}

El objetivo del presente artículo es conocer las diferentes herramientas tecnológicas que permiten una virtualidad en la educación, ya que debido al contexto que nos tocó enfrentar por la COVID-19, originó que los estudiantes busquen nuevas formas de aprender y los maestros nuevos desafíos que afrontar, por ello es necesario tener un soporte tecnológico para poder interactuar y aplicar las herramientas digitales haciendo de las aulas convencionales espacios virtuales, que permitan continuar con el aprendizaje, esto conlleva a los maestros a estar en constante aprendizaje y ver las mejores estrategias para sus estudiantes ya que esta modalidad también puede ser un elemento distractor para el estudiantes, por eso es necesario mantener la atención y motivación del estudiantes en todo momento, para lograr que sus aprendizajes sean similares o mejores a los obtenidos cuando la educación era de manera presencial. La metodología utilizada fue la revisión de variados artículos de investigaciones de las bases de datos de Google Académico, 
Scopus, EBSCO y ProQuest, obteniéndose 20 artículos publicados en revistas indexadas, realizadas en dos momentos específicos de búsqueda de acuerdo con las variables estudiadas entre los periodos de enero 2015 a diciembre 2020. Teniendo como resultado que son de vital importancia las herramientas tecnológicas en la virtualidad educativa, ya que genera espacios de aprendizajes colaborativos y fortalecen las capacidades cognitivas de los estudiantes.

Palabras clave: Herramientas tecnológicas, virtualidad en la educación, espacios digitales. 


\title{
Technological tools for a virtuality in education
}

\begin{abstract}
The objective of this article is to know the different technological tools that allow virtuality in education, since due to the context that we had to face due to COVID-19, it caused students to seek new ways of learning and teachers new challenges to face. Therefore, it is necessary to have technological support to be able to interact and apply digital tools, making conventional classrooms virtual spaces that allow learning to continue, this leads teachers to be constantly learning and see the best strategies for their students. since this modality can also be a distracting element for the students, that is why it is necessary to maintain the attention and motivation of the students at all times, to ensure that their learning is similar or better than that obtained when the education was face-to-face. The methodology used was the review of various research articles from the Google Scholar, Scopus, EBSCO and ProQuest databases, obtaining 20 articles published in indexed journals, carried out at two specific search times according to the variables studied between the periods. from January 2015 to December 2020.

Having as a result that technological tools in educational virtuality are of vital importance, since it generates collaborative learning spaces and strengthens the cognitive abilities of students.
\end{abstract}

Keywords: technological tools, virtuality in education, digital spaces.

Artículo recibido: 15 enero 2022 Aceptado para publicación: 08 febrero 2022 Correspondencia: mruizma@ucvvirtual.edu.pe Conflictos de Interés: Ninguna que declarar 


\section{INTRODUCCIÓN}

Estos dos últimos años la educación ha cambiado drásticamente tanto para estudiantes como para docentes, pasando de utilizar las herramientas tecnológicas como instrumentos de diversión y entretenimiento a utilizarlo en el campo educativo guiados por los docentes para la correcta utilización de estas herramientas, quienes los orientan en la adecuada búsqueda de información que los ayudará a discernir qué información es relevante e importante y cual no es necesaria, así de esta manera poder fomentar sus habilidades cognitivas y mejorar su rendimiento académico. Actualmente existen nuevos retos en los modelos formativos en la educación, siendo uno de estos retos el aprendizaje virtual, donde el conocimiento es obtenido con apoyo de las tecnologías a través de algunos dispositivos móviles como celulares, tabletas o laptops todos ellos con conexión a internet. La combinación de la investigación, el conocimiento y la innovación apoyados en las Tic permite poner en evidencia las transformaciones en la práctica pedagógica la cual facilita avanzar en el proceso formativo (Pérez, 2018).

Domínguez et al. (2017) nos comentan que es esencial una formación e información correcta de las herramientas tecnológicas, haciendo primero una reflexión crítica del magisterio quienes son los que van a orientar a los estudiantes en la forma apropiada de usar las tecnologías y a la vez van a prevenir cualquier amenaza que implica la utilización de estas herramientas especialmente la de las redes sociales. Para esto, es necesario invertir en capacitar a los docentes para que tengan las condiciones y competencias necesarias utilizándose de manera reflexiva y analítica.

El proceso autónomo: comprendida como el estudiante es libre e independiente para mejorar el aprendizaje virtual; requiere planificar su tiempo, ser disciplinados al estudiar priorizando actividades, siendo creativos, no guardarse lo brindado en la sesión, ya que el internet nos brinda una gama de recursos para poder adecuarlos y apropiarnos de ellos, finalmente el esfuerzo mental para preservar una buena actitud y no quedarse en el intento. En esta investigación se pone en evidencia la falta de equidad socioeducativas de los estudiantes, para esto, es fundamental profundizar en la percepción de las condiciones y las potenciales consecuencias que conllevan, para el sistema educativo. (Expósito y Marsollier, 2020).

Juanes et al. (2020) nos comentan En contexto actual que nos ha tocado vivir ante la pandemia, además de cumplir cuarentena obligatoria en algunos países, es imposible 
continuar con actividades presenciales con estudiantes, por lo que requiere el uso de metodologías que generen una interacción directa con los mismos, es por ello que se debe buscar metodologías y estrategias virtuales que permitan continuar con la labor pedagógica. Las tecnologías de información y comunicación (TIC), son herramientas tecnológicas interactivas que hoy en día permiten su desarrollo, mediante diversas estrategias en los actividades de entorno virtual entre maestros y discentes, que conlleven a capturar en todo momento su atención, mediante la World Wide Web (WWW) permite acceder a correos electrónicos, bibliotecas digitales, chats, foros, uso de recursos tecnológicos como Apps, videoconferencias de diferentes formas: conferencia, seminario y el teletrabajo, desde cada espacio de los estudiantes una de las ventajas de las TIC es que nos permiten acceder a miles de maestrías, especializaciones, carreras universitarias y cursos de modalidad virtual, a nivel mundial, permitiendo que cada vez más personas, accedan a la educación; donde se debe tener en cuenta las formas y estilos de aprendizajes de cada persona, generando en ellos nuevos retos del aprendizaje virtual. (Castellanos, 2021)

\section{ESTRATEGIAS METODOLÓGÍCAS}

El presente trabajo fue realizado revisando variadas investigaciones sobre el tema propuesto, a través de diferentes bases de datos como Google Académico, Scopus, EBSCO y Proquest con el fin de conceptualizar, definir conceptos importantes, sintetizar y evidenciar metodologías utilizadas previamente y distinguir los vacíos en investigación sobre el tema tratado, teniendo en cuenta procedimientos y técnicas de organización.

La metodología estuvo orientada a una revisión sistemática y analítica del tema tratado.

La búsqueda de la información se determinó en 5 años, entre el 2017 y 2021, considerando que, dado a la situación actual en la que vivimos desde hace dos años, los ambientes educativos virtuales han incrementado extremadamente. La indagación de artículos se desarrolló entre octubre de 2021 y enero del 2022.

\section{RESULTADOS}

La búsqueda de los artículos bibliográficos muestra los resultados de las variables de estudio de la investigación, teniendo como referente la búsqueda de artículos científicos publicados entre 2017 y 2021, así como, también los nombres de los autores. En la tabla 1 se puede observar lo anteriormente mencionado: 
Tabla 1: Variables de estudio

\begin{tabular}{|c|c|c|c|c|}
\hline $\mathbf{N}^{\mathbf{o}}$ & Tema & Autor & Año & Revista \\
\hline 1 & Educar la virtualidad & $\begin{array}{l}\text { Domínguez } \\
\text { Fernández, G., } \\
\text { Martínez Jaén, } \\
\text { A., \& Ceballos } \\
\text { García, M. J }\end{array}$ & 2017 & $\begin{array}{l}\text { Pixel-Bit. Revista de } \\
\text { Medios y Educación }\end{array}$ \\
\hline 2 & $\begin{array}{l}\text { Educación virtual un } \\
\text { nuevo desafío. }\end{array}$ & Pérez, C & 2018 & Revistas.sena.edu.com \\
\hline 3 & 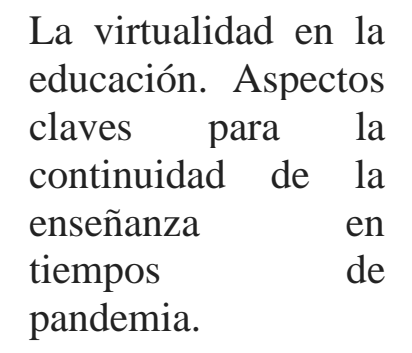 & $\begin{array}{l}\text { Juanes } \\
\text { Giraud, B. } \\
\text { Y., Munévar } \\
\text { Mesa, O. R., } \\
\text { \& Cándelo } \\
\text { Blandón, H }\end{array}$ & 2020 & Scielo \\
\hline 4 & $\begin{array}{l}\text { Virtualidad y } \\
\text { educación en tiempos } \\
\text { de COVID-19. Un } \\
\text { estudio empírico en } \\
\text { Argentina }\end{array}$ & $\begin{array}{l}\text { Expósito, C. } \\
\text { D., \& } \\
\text { Marsollier, } \\
\text { R. G. }\end{array}$ & 2020 & $\begin{array}{l}\text { Educación } \\
\text { humanismo }\end{array}$ \\
\hline 5 & $\begin{array}{l}\text { Ventajas y retos de la } \\
\text { virtualidad en la } \\
\text { educación. }\end{array}$ & $\begin{array}{l}\text { Castellanos, } \\
\text { A. A. }\end{array}$ & 2021 & $\begin{array}{l}\text { Revista Seres y Saberes, } \\
9(1) .\end{array}$ \\
\hline 6 & $\begin{array}{l}\text { Elementos clave de } \\
\text { la virtualidad en la } \\
\text { educación superior }\end{array}$ & $\begin{array}{l}\text { Calderón- } \\
\text { Meléndez, } \\
\text { A. }\end{array}$ & 2020 & $\begin{array}{lrr}\text { Revista } & \text { Electrónica } \\
\text { Calidad } & \text { en } & \text { la } \\
\text { Educación } & & \end{array}$ \\
\hline 7 & $\begin{array}{lr}\text { Educación } & \text { virtual: } \\
\text { oportunidad } & \text { para } \\
\text { «aprender } & \text { a } \\
\text { aprender" } & \end{array}$ & Sanabria, I. & 2020 & Análisis Carolina \\
\hline 8 & $\begin{array}{l}\text { Virtualidad como } \\
\text { herramienta de apoyo a } \\
\text { la presencialidad: } \\
\text { Análisis desde la } \\
\text { mirada estudiantil. }\end{array}$ & $\begin{array}{l}\text { Carmona, C. } \\
\text { V., \& } \\
\text { Mancero, P. } \\
\text { C. B. }\end{array}$ & 2020 & $\begin{array}{l}\text { Revista de Ciencias } \\
\text { sociales }\end{array}$ \\
\hline 9 & $\begin{array}{lll}\text { Estrategias } & & \text { de } \\
\text { enseñanza } \\
\text { virtualidad. }\end{array}$ & $\begin{array}{l}\text { Szpiniak, A. } \\
\text { F. }\end{array}$ & 2020 & $\begin{array}{l}\text { Universidad Nacional } \\
\text { de Río Cuarto. }\end{array}$ \\
\hline
\end{tabular}


Ruiz Sánchez y otros...

\begin{tabular}{|c|c|c|c|c|}
\hline 10 & $\begin{array}{l}\text { Competencias digitales } \\
\text { y su integración con } \\
\text { herramientas } \\
\text { tecnológicas } \\
\text { educación superior. }\end{array}$ & $\begin{array}{l}\text { Vargas- } \\
\text { Murillo, G. }\end{array}$ & 2019 & Misceláneas \\
\hline 11 & $\begin{array}{l}\text { Educación a distancia } \\
\text { en tiempos de COVID- } \\
\text { 19: Análisis desde la } \\
\text { perspectiva de los } \\
\text { estudiantes } \\
\text { universitarios }\end{array}$ & $\begin{array}{l}\text { Pérez- } \\
\text { López, Eva; } \\
\text { Vázquez } \\
\text { Atochero, } \\
\text { Alfonso; } \\
\text { Cambero } \\
\text { Rivero, } \\
\text { Santiago }\end{array}$ & 2021 & $\begin{array}{l}\text { RIED. Revista } \\
\text { Iberoamericana de } \\
\text { Educación a Distancia }\end{array}$ \\
\hline 12 & $\begin{array}{l}\text { Desde la educación a } \\
\text { distancia al e-Learning } \\
\text { emergencia, evolución } \\
\text { y consolidación }\end{array}$ & $\begin{array}{l}\text { Pablo } \\
\text { Rivera } \\
\text { Vargas, } \\
\text { Cristina } \\
\text { Alonso } \\
\text { Cano, Juana } \\
\text { María } \\
\text { Sancho Gil }\end{array}$ & 2017 & Dialnet \\
\hline 13 & $\begin{array}{l}\text { El desafío de la } \\
\text { presencialidad a la } \\
\text { virtualidad en la } \\
\text { educación superior en } \\
\text { tiempos de pandemia. }\end{array}$ & $\begin{array}{l}\text { Ruiz- } \\
\text { Aquino, M. }\end{array}$ & 2020 & Desafíos \\
\hline 14 & $\begin{array}{l}\text { Educación a distancia: } \\
\text { transformación de los } \\
\text { aprendizajes }\end{array}$ & $\begin{array}{l}\text { Liliana } \\
\text { Yonué } \\
\text { Covarrubias } \\
\text { Hernández }\end{array}$ & 2021 & $\begin{array}{l}\text { Telos Revista } \\
\text { Científica Arbitrada. }\end{array}$ \\
\hline 15 & $\begin{array}{l}\text { Representaciones } \\
\text { sociales de la } \\
\text { educación a distancia } \\
\text { durante la pandemia } \\
\text { por COVID-19 }\end{array}$ & $\begin{array}{l}\text { Berenice } \\
\text { Jaime } \\
\text { Romero }\end{array}$ & 2022 & $\begin{array}{l}\text { CPU-e Revista de } \\
\text { investigación educativa }\end{array}$ \\
\hline
\end{tabular}




\begin{tabular}{|c|c|c|c|c|}
\hline 16 & 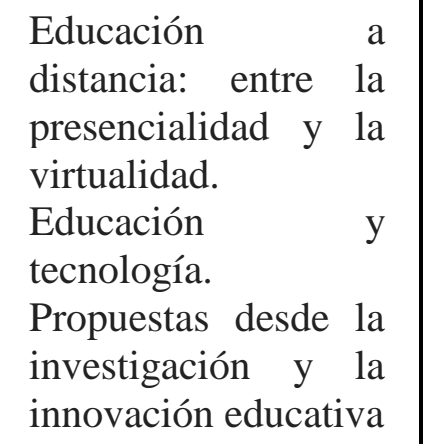 & $\begin{array}{l}\text { Lancheros, } \\
\text { M. E. P., \& } \\
\text { González, } \\
\text { M. J. C. }\end{array}$ & (2017). & Acta Académica \\
\hline 17 & $\begin{array}{l}\text { Lo que la pandemia nos } \\
\text { enseñó sobre la } \\
\text { educación a distancia }\end{array}$ & $\begin{array}{l}\text { Mendoza } \\
\text { Castillo, } \\
\text { Lucía }\end{array}$ & 2020 & $\begin{array}{l}\text { Revista } \\
\text { Latinoamericana de } \\
\text { Estudios Educativos } \\
\text { (México }\end{array}$ \\
\hline 18 & $\begin{array}{l}\text { Educación remota de } \\
\text { emergencia, } \\
\text { virtualidad } \\
\text { desigualdades: } \\
\text { pedagogía en tiempos } \\
\text { de pandemia. } 593 \\
\text { CEIT, 5(5), 98-107. }\end{array}$ & López, L. & 2020). & $\begin{array}{l}\text { Digital } \\
\text { Publisher }\end{array}$ \\
\hline 19 & $\begin{array}{l}\text { Hacia una pedagogía en } \\
\text { la virtualidad: } \\
\text { vincularidad en tiempos } \\
\text { de pandemia?. }\end{array}$ & $\begin{array}{l}\text { Bobadilla, } \\
\text { M. J., Miño } \\
\text { Vargas, D., } \\
\text { \& Rago, M. }\end{array}$ & (2020). & RED SOCIALES \\
\hline 20 & $\begin{array}{l}\text { Ejecución de una clase } \\
\text { dentro del aula y en la } \\
\text { virtualidad. } \\
\text { Revista } \\
\text { Arbitrada } \\
\text { Interdisciplinaria } \\
\text { Koinonía, 5(1), } \\
410 .\end{array}$ & $\begin{array}{l}\text { González, } \\
\text { R. C. V., } \\
\text { Herrera, D. } \\
\text { G. G., } \\
\text { Clerque, S. } \\
\text { E. M., \& } \\
\text { Álvarez, J. } \\
\begin{array}{l}\text { C. E. }\end{array}\end{array}$ & (2020). & $\begin{array}{l}\text { Revista Arbitrada } \\
\text { Interdisciplinaria } \\
\text { KOINONIA }\end{array}$ \\
\hline
\end{tabular}

Fuente: Base de datos Google Académico, EBSCO, Proquest, Scopust

Cuando hablamos de una educación virtual no se trata solamente de elaborar videos y socializarlos con los estudiantes, sino que es necesario contar con las habilidades y destrezas en este campo para poder lograr con el objetivo previsto, además de tener las capacidades y competencias necesarias para brindar los contenidos pertinentes relacionadas al área. Siendo necesario una buena planificación de lo que se logrará con los estudiantes, teniendo en cuenta una evaluación diagnóstica para conocer su situación de cómo se encuentran y de acuerdo con eso priorizar los contenidos que se trabajaran, 
no podemos dejar de lado los ritmos y estilos de aprendizaje de cada estudiante. El docente debe ser creativo y estar capacitado constantemente en entornos virtuales para poder captar en todo momento la atención y motivación del estudiante, ya que aprender en esta modalidad virtual conlleva a que los estudiantes se distraigan con facilidad y no puedan seguir una secuencia planificada por el docente. por eso es necesario que seleccione los entornos y herramientas adecuadas para lograr el propósito previsto (Calderón, 2020).

Sanabria (2020) afirma que las experiencias impartidas conllevan a formularnos la siguiente interrogante: ¿cómo puede el docente interactuar con los estudiantes en entornos virtuales para lograr que desarrollen un pensamiento crítico, creativo, reflexivo y con habilidades metacognitivas, que le ayude a “aprender a aprender”? No es fácil responder a esta pregunta, ya que esto conlleva al análisis de cómo se desarrollaría una experiencia de este tipo, donde el estudiantes participe de manera activa elaborando mapas mentales, creando historietas, juegos interactivos, utilizando aplicaciones para resolver problemas matemáticos y que a su vez permita que vaya movilizando diferentes capacidades para finalmente aplicarlas en la resolución de problemas en su vida diaria.

Camona y Mancero (2020) manifiestan que los estudiantes perciben a la virtualidad como una ayuda dinámica, participativa e innovadora el cual permite realizar trabajos colaborativos entre ellos, facilitando la comunicación en los grupos de trabajo y una mejor interacción con sus docentes. En esta modalidad también se presentan algunas limitaciones personales como el no contar con aparatos tecnológicos y servicio de internet en casa siendo para ellos un obstáculo para desarrollar sus actividades académicas.

Szpiniak, (2020) nos comenta que no debemos olvidar que la combinación de clases virtuales es una magnífica forma de alcanzar las sugerencias pedagógicas, lo que significa que podemos usar la virtualidad para sustituir la presencialidad, entendiendo que la virtualidad jamás reemplazará la presencialidad, especialmente en áreas donde es necesario el trabajo de campo. En este sentido, no debemos caer en los mismos errores de trabajar igual de forma virtual que presencial, ya que son dos formas diferentes de trabajar, ya que no tenemos la certeza de que nuestros estudiantes tienen las mismas oportunidades de conectividad en los horarios y fechas programadas por el docente. Una de las mejores estrategias virtuales es optimizar las herramientas sincrónicas las cuales 
están disponibles las 24 horas y tener activas las plataformas virtuales de forma permanente.

Las habilidades digitales en esta era, son todas las habilidades y destrezas básicas que los maestros, estudiantes y demás profesionales requieren para fortalecer y potenciar sus capacidades de enseñar, aprender e investigar. Por otra parte, los usuarios deben identificar y elegir los recursos tecnológicos que no requieren de un pago o uno que esté acorde a sus necesidades. Finalmente, otro aspecto esencial de la competencia digital es que fomenta entornos virtuales científicos colaborativos y flexibles que, además de generar conocimiento, permitan a los usuarios estar al día con las tecnologías y los nuevos medios. (Vargas, 2019).

Pérez et al. (2021) sostienen que los estudiantes universitarios de manera presencial y a distancia de las universidades han implementado estrategias para esta etapa de confinamiento por la COVID-19. siendo su principal propósito analizar el contexto tanto personal como familiar en la igualdad digital, debiendo conocer el modelo de la enseñanza recibida. Esta investigación resulta del estudio mixto teniendo un alcance descriptivo combinando el método cualitativo y cuantitativo. aplicando un cuestionario a los estudiantes de la universidad, también se realizaron entrevistas online a las autoridades de la universidad. Resultando que los estudiantes provienen de familias de nivel formativo bajo concluyendo que tienen menos oportunidades en el uso de herramientas digitales. Las clases virtuales se han realizado a través de archivos subidos al campus virtual y comunicación asincrónicas. En consecuencia, se concluye que la universidad debe procurar modelos colaborativos y que estén fijados en el estudiante.

Rivera et al. (2017) manifiestan si existe una relación directa del e-Learning con lo que conocemos de educación a distancia convencional. Haciendo una búsqueda minuciosa sobre investigaciones relacionadas al respecto, se pudo determinar que ambas modalidades tienen principios y propósitos distintos. Institucionalmente se puede observar un cambio en ambos conceptos, teniendo relación y vínculo. Con el fin de aclarar este punto, se realizó el presente estudio donde se afrontó esta diferencia, ahondando en estos aspectos y componentes que se terminó por componer lo que hoy sabemos por eLearning. abordando tres dimensiones principales: Las diferentes modalidades que se tiene en la formación de educación superior, el proceso de desarrollo a distancia y su concepto tradicional, y el cambio que genera la incorporación y aplicación de manera 
paulatina de las TIC y por último los principales conceptos y características del eLearning.

Reflexionar el nuevo contexto en la que la comunidad estudiantes y maestros enfrentan luego enfrentar por la pandemia a la que la OMS declaró la COVID-19, como una nueva enfermedad contagiosa que acaparó mundialmente, generando espacios virtuales para una nueva forma de aprendizajes de la mano con el temor y las diferencias sociales que se presenta de forma generalizada. Respaldado en la meditación y el estudio teórico de algunos estudios, se observa en América Latina se ha dado continuidad a los procesos educativos teniendo en cuenta la digitalidad aunque los gobiernos no le dan el principal interés Siendo necesario la motivación y la concientización en los agentes educativos que estamos obligados a aportar en esta nueva forma de aprender sin condición social, generando empatía para poder entender que un gran número no cuenta con herramientas tecnológicas y en algunos casos no saben cómo interactuar con ellas. Es por ello que debemos tomar conciencia y aportar para seguir avanzando en estos nuevos retos. (Covarrubias, 2021)

Estudios realizados tienen como propósito determinar las representaciones sociales que conocen los estudiantes de bachillerato tecnológico sobre la educación a distancia en tiempo de pandemia. Se admitió el enfoque estructural por Abric (2001). Para obtener la información se hizo a través de una encuesta en línea, tomando un muestreo no probabilístico. Los resultados obtenidos fueron que para los estudiantes la educación a distancia genera estrés y acumulo de trabajos. Llegando a la conclusión que la aproximación a las representaciones sociales de la educación a distancia genera un aporte importante, ya que se observa el interés que esta generando en los estudiantes y la necesidad de incluirla en el proceso de enseñanza aprendizaje (Jaime, 2021)

Mendoza (2020) Actualmente atravesamos una situación complicada, que ha llevado a que los agentes educativos se ven obligados buscar y utilizar estrategias para poder continuar los procesos de aprendizaje de manera remota, en consecuencia, estas estrategias no son las mismas a los proyectos educativos de educación a distancia. En este aspecto se hace una planeación, el tiempo para ejecutarlo, los temas a trabajar y las horas asignadas al mismo, que en este caso corresponde a los presencial. Los maestros en el aula conocen a sus estudiantes, sabe su forma de aprender, sus limitaciones y sus fortalezas, interactuando activamente con el ellos y de acuerdo a eso los evalúa. 
López (2020) manifiesta que se pudo observar importantes problemáticas en función a la educación responsable en el contexto de la Covid-19. Siendo necesaria la transición de la educación presencial a las modalidades virtuales y remotas. La enseñanza tradicional ha tenido una serie de controversia con respecto a su naturaleza. se puede observar a lo largo de la historia las diferentes modalidades teniendo en cuenta las generaciones y que conllevan a formas virtuales de aprendizajes. Siendo el acceso una traba y la calidad que se brinda en la educación se ven afectados con la pandemia mundial que se vive hoy en día. Esto lleva a que debemos vernos en la necesidad de afrontar esos cambios que ha generado el contexto y asumirlos responsablemente para poder contribuir a la educación. Bobadilla et al. (2020) manifiestan que, al iniciar la pandemia, nuestras vidas se han visto afectadas en todos los aspectos de nuestra vida cotidiana. Sin imaginar que nuestras vidas darían un giro inesperado e hizo que tengamos que ver nuevas formas de aprender (estudiantes) y de enseñar (docentes). En este nuevo reto educativo los docentes asumen un nuevo rol agenciando de nuevas estrategias de enseñanza concernientes a los soportes tecnológicos y digitales que permitan que podamos continuar con nuestra labor educativa. Haciendo uso de diferentes aplicaciones que nos ofrecen la tecnología generando en algunas ocasiones desconcierto ante situaciones nuevas, ya que algunos maestros no tienen la facilidad para interactuar con ellos creando una desigualdad en algunos casos, por otro lado, las dificultades al acceso y herramientas tecnológicas que agudizan más la problemática. Compartiremos algunas estrategias pedagógicas que fueron desarrollándose en el transcurso del estudio que impliquen un mejor desenvolvimiento tanto para los estudiantes como para los maestros, siempre y cuando exista también la predisposición a asumir estos desafíos para mejorar en nuestro quehacer diario.

González et al. (2020). concluyeron que al ejecutar los tres momentos de la sesión se hace un análisis exhaustivo de todo lo que conlleva este aspecto, siendo necesario tener en cuenta el ambiente, ya que eso permite que el alumno mantenga la motivación e interés desde el inicio hasta el final de la clase, eso genera que el estudiante edifique sus propios conocimientos y los convierte en aprendizajes significativos. También se puede determinar que la aplicación de los tres momentos se puede desarrollar dentro de un aula modificando algunos aspectos particulares, si se aplica a la virtualidad se vuelve tedioso ya que implica que se tiene que disminuir los tiempos de trabajo, no existe un contacto 
físico directos con el estudiante y no se puede observar con precisión sus avances en el logro de aprendizajes. En ese contexto la tecnología no sólo es un apoyo para un determinado momento de la sesión de clase, sino es fundamental en el desarrollo de todo el proceso. Dentro de lo obtenido se puede resaltar la importancia de los enfoques pedagógicos, en la enseñanza aprendizaje, siendo una pieza indispensable en el entorno educativo. ya que están estrechamente ligados con el conocimiento y la pedagogía. También se concluye que el uso de la tecnología en los maestros no sólo es suficiente para poder desenvolverse en entornos virtuales, también puede estar afectado por el escaso conocimiento de la pedagogía y en otro caso la carencia de capacitación en entornos virtuales por parte de los maestros.

\section{DISCUSIÓN}

Al analizar los resultados, podemos determinar que las herramientas tecnológicas son de vital importancia en la aplicación de de una educación virtual ya que los estudiantes se motivan mucho más teniendo un entorno innovador para aprender.

También se puede observar que los docentes se vieron en la necesidad de aprender e interactuar nuevas formas de enseñar, haciendo uso de nuevas metodologías, herramientas y espacios generados por ellos mismos para continuar impartiendo sus clases buscando un ambiente adecuado para poder aproximarse a un aula real.

Con respecto a los estudiantes se adecuaron rápidamente a este nuevo sistema de la virtualidad ya que utilizan aplicaciones para poder desarrollar los trabajos asignados por sus maestros, se relacionan fácilmente con sus pares para realizar trabajos colaborativos haciendo uso de espacios en línea.

Las herramientas tecnológicas son sin duda alguna un soporte para los maestros y estudiantes para poder continuar con la enseñanza aprendizaje ante situaciones adversas, generando espacios de interaprendizaje continuos y donde la información se torna de fácil acceso y confiable.

\section{CONCLUSIONES}

El uso de las herramientas tecnológicas en la virtualidad en la educación incide favorablemente tanto en estudiantes como en maestros ya que generan un interaprendizaje y aprendizaje continuo, generando conocimientos en diferentes áreas, siendo un soporte significativo en la educación. 
Las herramientas tecnológicas y la educación virtual están relacionadas generando nuevos desafíos en los maestros en la parte pedagógica, logrando experiencias innovadoras y muy significativas en los estudiantes, siempre y cuando tome conciencia que la virtualidad no solo es la transferencia de la información, sino que va más allá, teniendo que analizar y sintetizar lo que tenemos para utilizarlo en nuestro quehacer diario.

Las Instituciones que brindan servicio educativo deberán establecer las mejores condiciones para generar estos espacios de aprendizaje teniendo en cuenta desde un buen entorno tecnológico y el acceso a internet para garantizar que el aprendizaje que se brinde sea significativo.

\section{REFERENCIAS BIBLIOGRAFICAS}

Bobadilla, M. J., Miño Vargas, D., \& Rago, M. (2020). Hacia una pedagogía en la virtualidad: $i$ vincularidad en tiempos de pandemia?

Calderón-Meléndez, A. (2020). Elementos clave de la virtualidad en la educación superior. Revista Electrónica Calidad en la Educación Superior, 11(2), 80-104.

Carmona, C. V., \& Mancero, P. C. B. (2020). Virtualidad como herramienta de apoyo a la presencialidad: Análisis desde la mirada estudiantil. Revista de Ciencias sociales, 26(1), 219-232.

Castellanos, A. A. (2021). VENTAJAS Y RETOS DE LA VIRTUALIDAD EN LA EDUCACIÓN. Revista Seres y Saberes, 9(1).

Castillo, L. M. (2020). Lo que la pandemia nos enseñó sobre la educación a distancia. Revista Latinoamericana de Estudios Educativos (México), 50, 343-352.

Domínguez Fernández, G., Martínez Jaén, A., \& Ceballos García, M. J. (2017). Educar la virtualidad. Pixel-Bit. Revista de Medios y Educación, 50, 187-199.

Expósito, C. D., \& Marsollier, R. G. (2020). Virtualidad y educación en tiempos de COVID-19. Un estudio empírico en Argentina.

González, R. C. V., Herrera, D. G. G., Clerque, S. E. M., \& Álvarez, J. C. E. (2020). Ejecución de una clase dentro del aula y en la virtualidad. Revista Arbitrada Interdisciplinaria Koinonía, 5(1), 392-410.

Hernández, L. Y. C. (2021). Educación a distancia: transformación de los aprendizajes. Telos: revista de Estudios Interdisciplinarios en Ciencias Sociales, 23(1), 150160. 
Juanes Giraud, B. Y., Munévar Mesa, O. R., \& Cándelo Blandón, H. (2020). La virtualidad en la educación. Aspectos claves para la continuidad de la enseñanza en tiempos de pandemia. Conrado, 16(76), 448-452.

Lancheros, M. E. P., \& González, M. J. C. (2017). Educación a distancia: entre la presencialidad y la virtualidad. EDUcación y TECnología. Propuestas desde la investigación y la innovación educativa, 39.

López, L. (2020). Educación remota de emergencia, virtualidad y desigualdades: pedagogía en tiempos de pandemia. 593 digital Publisher CEIT, 5(5), 98-107.

Pérez, C. A. C. (2018). Educación virtual un nuevo desafío. Reto, 6(1), 11-19.

Pérez-López, E., Atochero, A. V., \& Rivero, S. C. (2021). Educación a distancia en tiempos de COVID-19: Análisis desde la perspectiva de los estudiantes universitarios. RIED. Revista Iberoamericana de Educación a Distancia, 24(1), 331-350.

Romero, B. J. (2022). Representaciones sociales de la educación a distancia durante la pandemia por COVID-19. CPU-e, Revista de Investigación Educativa, (34).

Ruiz-Aquino, M. (2020). El desafío de la presencialidad a la virtualidad en la educación superior en tiempos de pandemia. Desafios, 11(1), e136-e136.

Sanabria, I. (2020). Educación virtual: oportunidad para «aprender a aprender». Análisis Carolina, (42), 1.

Szpiniak, A. F. (2020). Estrategias de enseñanza en la virtualidad. Universidad Nacional de Río Cuarto. Recuperado de: https://www. evelia. unrc. edu. ar/ensenaryAprenderEnLaVirtualidad/2020/12/02/estrategias-de-ensenanza-enla-virtualidad.

Vargas, P. R., Cano, C. A., \& Gil, J. M. S. (2017). Desde la educación a distancia al eLearning: emergencia, evolución y consolidación. Revista educación y tecnología, (10), 1-13.

Vargas-Murillo, G. (2019). Competencias digitales y su integración con herramientas tecnológicas en educación superior. Cuadernos Hospital de clínicas, 60(1), 8894. 